\title{
The Genetic Base for Peanut Height-Related Traits Revealed by a Meta-Analysis
}

\author{
Juan Wang ${ }^{1}$, Caixia Yan ${ }^{1}$, Dachuan Shi ${ }^{2}{ }^{\circledR}$, Xiaobo Zhao ${ }^{1}$, Cuiling Yuan ${ }^{1}$, Quanxi Sun ${ }^{1}{ }^{\circledR}$, Yifei Mou ${ }^{1}$, \\ Haoning Chen ${ }^{1}$, Yuan $\mathrm{Li}^{3, *}$, Chunjuan $\mathrm{Li}^{1, *}$ and Shihua Shan ${ }^{1, *}$ \\ 1 Shandong Peanut Research Institute, Qingdao 266100, China; wangjuan_1984@163.com (J.W.); \\ cxyan335@sina.com (C.Y.); zhaoxiaoboqd@126.com (X.Z.); yuancl1982@163.com (C.Y.); \\ squanxi@163.com (Q.S.); yifeimou1123@163.com (Y.M.); chny.312@163.com (H.C.) \\ 2 Qingdao Academy of Agricultural Sciences, Qingdao 266100, China; catchabreak@163.com \\ 3 Computational Biology and Biological Physics, Astronomy and Theoretical Physics, Lund University, \\ 22100 Lund, Sweden \\ * Correspondence: Yuan.Li@thep.lu.se (Y.L.); peanutlab@163.com (C.L.); shansh_spri@163.com (S.S.); \\ Tel.: +46-(0)73-5854-860 (Y.L.); +86-(0)532-8762-9308 (C.L.); +86-532-8762-9307 (S.S.)
}

Citation: Wang, J.; Yan, C.; Shi, D.; Zhao, X.; Yuan, C.; Sun, Q.; Mou, Y.; Chen, H.; Li, Y.; Li, C.; et al. The Genetic Base for Peanut Height-Related Traits Revealed by a Meta-Analysis. Plants 2021, 10, 1058. https://doi.org/10.3390/

plants10061058

Academic Editor: Adnane Boualem

Received: 19 April 2021

Accepted: 17 May 2021

Published: 25 May 2021

Publisher's Note: MDPI stays neutral with regard to jurisdictional claims in published maps and institutional affiliations.

Copyright: (C) 2021 by the authors Licensee MDPI, Basel, Switzerland. This article is an open access article distributed under the terms and conditions of the Creative Commons Attribution (CC BY) license (https:// creativecommons.org/licenses/by/ $4.0 /)$.

\begin{abstract}
Peanut (Arachis hypogaea L.) is an important oilseed crop worldwide, and peanut height has been shown to be closely related to yield, therefore a better understanding of the genetic base of plant height-related traits may allow us to have better control of crop yield. Plant height-related traits are quantitative traits that are genetically controlled by many genes, and distinct quantitive trait loci (QTLs) may be identified for different peanut accessions/genotypes. In the present study, in order to gain a more complete picture of the genetic base for peanut height-related traits, we first make use of the high quality NGS sequence data for 159 peanut accessions that are available within our research groups, to carry out a GWAS study for searching plant height-related regions. We then perform a literature survey and collect QTLs for two plant height-related traits (Ph: peanut main stem height, and Fbl: the first branch length) from earlier related QTL/GWAS studies in peanut. In total, we find 74 and 21 genomic regions that are, associated with traits $\mathrm{Ph}$ and Fbl, respectively. Annotation of these regions found a total of 692 and 229 genes for, respectively, Ph and Fbl, and among those genes, 158 genes are shared. KEGG and GO enrichment analyses of those candidate genes reveal that Phand Fbl-associated genes are both enriched in the biosynthesis of secondary metabolites, some basic processes, pathways, or complexes that are supposed to be crucial for plant development and growth.
\end{abstract}

Keywords: peanut; plant height; secondary metabolites; meta-analysis

\section{Introduction}

As one important source of edible oil, the groundnut (Arachis hypogaea L.) is widely cultivated across warm temperate, subtropical, and tropical zones [1-3], with China being its largest producer and exporter [4]. Nevertheless, there is still a huge market demand for peanuts, which may be met by genetic improvement of its agronomic traits $[5,6]$. Peanut height has been shown to be closely related to yield, and co-localization of QTLs (Quantitative Trait Loci) for peanut height- and yield-related traits have also been reported [7-11], therefore, understanding the genetic base of plant height-related traits may allow us to have better control of crop yield. The plant height-related traits, such as plant main stem height (Ph), nodes on the main stem (Nsk), and the first branch length (Fbl), are quantitative traits, which are controlled by multiple loci and at the same time influenced by variable environmental factors $[7,12]$. Therefore, it is very challenging to explicitly address the genetic base of a plant height-related trait, nevertheless, efforts have been made by both QTL mapping as well as GWAS studies in recent years, which move us closer and closer towards this goal [7,9,13-17].

In the current study, we make use of the available NGS data for 159 of the 195 peanut accessions that were generated by our earlier yield-related trait-focused GWAS study [18] 
to further explore the genetic base of plant height-related traits. These NGS data together with phenotype data on three plant height-related traits that were collected from the same peanut accessions make it possible for us to perform GWAS analyses, from which candidate genomic regions have been identified for two of the studied traits. Meanwhile, we have also collected QTLs, for the same traits, from earlier QTL/GWAS analyses that together represent a high diversity of peanut genotypes. A summary of all the annotated genes within the candidate genomic regions from both the present GWAS and earlier QTL/GWAS analyses have helped us gain a more complete picture of the underlying genetic base for the two height-related traits.

\section{Results}

\subsection{Characterisation and Distribution of Genetic Variations of the Peanut Genome}

A total of 15,556 SNPs and 1561 InDels have been identified from the collected peanut genomes (Table S2), leading to a genome-wide SNP density of $6.13 \mathrm{SNPs} / \mathrm{Mb}$, with chromosomes Arahy.08 (4.24 SNPs/Mb) and Arahy.19 (8.77 SNPs/Mb) being, respectively, the sparsest and the densest. Most of the identified SNPs are found at intergenic regions $(90.12 \%)$, while the exonic, intronic, upstream, and downstream regions only account for $3.05 \%, 3.78 \%$, and $3.05 \%$ of the total SNPs, respectively. Similarly, the genome has a genome-wide InDel density of 0.61 InDels $/ \mathrm{Mb}$ with chromosomes Arahy.02 (0.39 InDels $/ \mathrm{Mb}$ ) and Arahy.08 (0.91 InDels/Mb) having, respectively, the sparsest and the densest InDels (Figure 1). Most of the identified InDels are also found at intergenic regions $(71.68 \%$ ), while the exonic, intronic, up- and down-stream regions only contain, respectively, $5.06 \%$, $13.84 \%$ and $9.42 \%$ of the total InDels. The genome-wide transition/transversion (Ts/Tv) ratio for the analyzed peanut genome data is 1.81 .

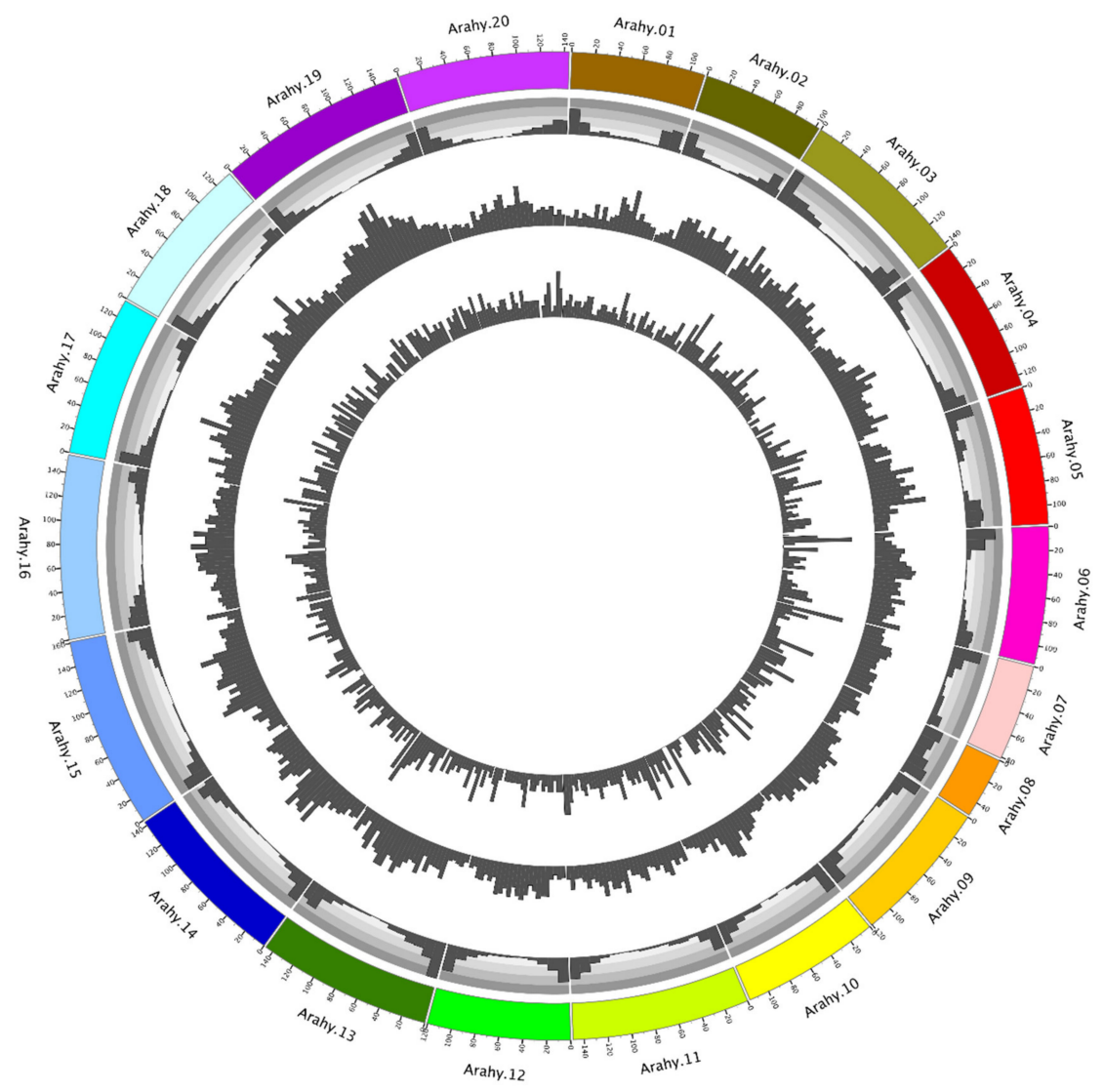

Figure 1. Genome map of peanut. The four layers from the outmost to the innermost, respectively, represent chromosomes, gene density, SNP density, and InDel density. 


\subsection{Phenotypic Correlation and Heritability for Different Traits}

A total of 159 peanut accessions have been phenotyped for their plant main stem height [Ph], the first branch length [Fbl], and the number of nodes on the main stem [Nsk] over three years at three different locations. All traits follow a normal distribution with low values of skew and kurtosis (Supplementary Materials Figures S2-S5). We observe an intermediate level of heritability $\left(H^{2}: 0.44-0.51\right)$ for all three traits, which are positively correlated with each other $(r$ : 0.55-0.64) (Table 1 and Table S3; Figure S6).

Table 1. Phenotypic Statistics of the Three Studied Plant Height Related Traits.

\begin{tabular}{ccccccccccccc}
\hline Trait & Sample & Mean & SD & SE & Median & Min & Max & Range & Skew & Kurtosis & CV $\%^{*}$ & $\boldsymbol{H}^{\mathbf{2}}$ \\
\hline Fbl & 1422 & 48.61386 & 13.4337 & 0.356243 & 48.2 & 11.6 & 87.8 & 76.2 & 0.22002 & -0.35783 & 27.63 & 0.513899 \\
Nsk & 1423 & 17.47671 & 4.318244 & 0.114473 & 17.4 & 5.2 & 30.4 & 25.2 & 0.037926 & -0.18738 & 24.71 & 0.443737 \\
Ph & 1417 & 35.078 & 10.65981 & 0.283181 & 34.0 & 6.6 & 67.8 & 61.2 & 0.450744 & -0.11826 & 30.39 & 0.473302 \\
\hline
\end{tabular}

NOTE: SD, standard deviation; $\mathrm{SE}$, standard error; $\mathrm{CV}$, coefficient of variance; $H^{2}$, broad-sense heritability.

\subsection{Genome-Wide Association Study (GWAS) in Peanut}

Genome-wide association analysis of the phenotypically characterized peanut accessions has been conducted to see if any of the acquired SNPs are associated with the studied plant height-related traits. For traits Ph and Fbl, tests with the MLM (K) and MLM (QK) models, especially MLM $(\mathrm{QK})$, produce $p$-values that deviate dramatically less from expectation than the $p$-values using the GLM and GLM (Q) models (QQ plots in Figure S7). However, for MLM (QK), the signal for a significant association is weak, especially the test $p$-values become larger than expected when it goes towards the $Q Q$ plot tip, suggesting possible overcorrection of confounding factors by the $Q$ and $K$ matrices [19]. Nevertheless, under the MLM (QK) model, we are still able to detect significantly associated peak SNPs for the studied traits by a significance level of $10^{-5}\left(1.1 \times 10^{-6} \sim 9.6 \times 10^{-6}\right)$. For $\mathrm{Ph}$, two of the identified associated peak SNPs are located on chromosome Arahy.02, three on Arahy.16, one on each of Arahy.19, and Arahy.20 (Figure 2). For Fbl that has a relatively higher heritability $\left(H^{2}=0.51\right)$ comparing to $\mathrm{Ph}$, only four associated peak SNPs have been identified, three from Arahy.16 and one on Arahy.18. It is worth noting that traits $\mathrm{Ph}$ and $\mathrm{Fb} 1$ share one associated peak SNPs on Arahy.16, Arahy.16:32726692, and even more interestingly, from the candidate genome region that is centered on this shared peak SNP, two genes (arahy.DT3E7E at Arahy.16:32705499-32708164; arahy.AG6W8J at Arahy.16:32708616-32709684) are annotated to be LRR receptor-like kinase. LRR receptor-like kinases are mostly involved in signal transduction and play an essential role in plant development and stress resistance [20-22]. No significant association has been found for the trait Nsk.
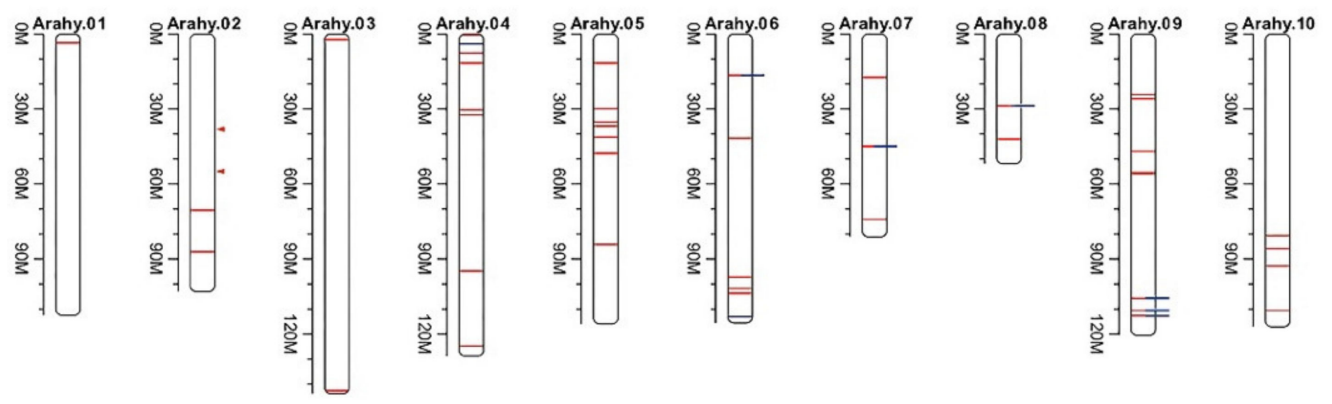

Figure 2. Conts. 

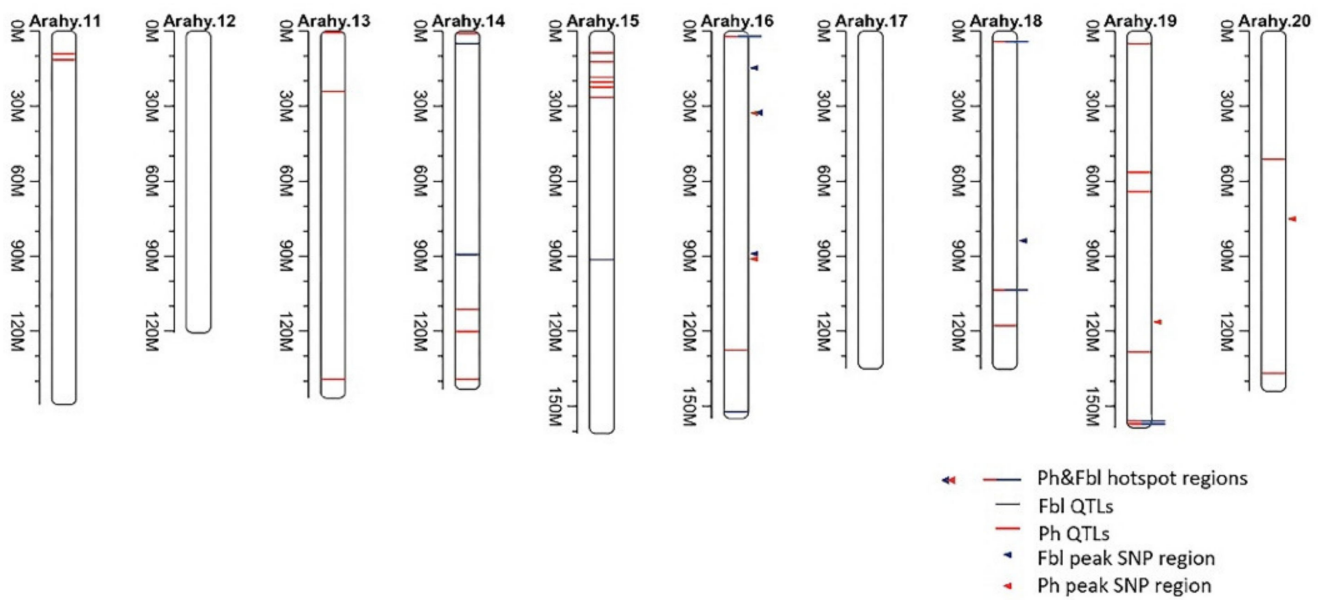

Figure 2. Quantitative trait loci (QTLs) identified to be associated with Ph and/or Fbl by both this and earlier studies. The blue lines represent the QTLs for Fbl identified from earlier studies while red for $\mathrm{Ph}$. The solid triangles point to the peak SNP regions that are identified to be associated with $\mathrm{Ph}$ (red) and/or Fbl by the present GWAS analysis.

\subsection{A Summary of QTLs, for Peanut Ph and Fbl, Identified from Both Present and Earlier Studies}

We have performed a thorough literature survey and found twelve QTL/GWAS studies that also looked at the same peanut phenotypic traits as we do though with different sets of peanut accessions (Table S4). These studies, together with the current GWAS study, have identified 74 and 21 genomic regions that are, respectively, associated with traits $\mathrm{Ph}$ and $\mathrm{Fbl}$ (Table S5; Figure 2). Annotation of these regions found a total of 692 and 229 genes for, respectively, $\mathrm{Ph}$ and $\mathrm{Fbl}$, among those genes, 158 genes are shared (Tables S5 and S6).

KEGG enrichment analysis found that the Ph-associated genes are enriched in four metabolic pathways (Brassinosteroid biosynthesis, Biosynthesis of secondary metabolites, beta-Alanine metabolism, and Ascorbate and aldarate metabolism) as well as one genetic information processing complex (Proteasome) (Figure 3). While GO enrichment analyses show that the candidate genes for $\mathrm{Ph}$ are enriched in the proteasome complex (the "Cellular Component" category), in the developmental process of regulating epidermis development (the "Biological Process" category), as well as in several catalytic activities (the "Molecular Function" category): oxidoreductase activity, endopeptidase activity, rRNA methyltransferase activity, oxidoreductase activity (acting on $\mathrm{CH}-\mathrm{OH}$ group of donors), plus three hydrolase activities (inositol tetrakisphosphate phosphatase activity, inositol trisphosphate phosphatase activity, and galactosidase activity) (Figure 4).

For $\mathrm{Fbl}$, its associated genes are over-represented in one KEGG genetic information processing complex (Spliceosome) and several KEGG metabolic pathways: Biosynthesis of secondary metabolites (Isoquinoline alkaloid biosynthesis; Tropane, piperidine, and pyridine alkaloid biosynthesis), Amino acid metabolism (Phenylalanine metabolism, and Tyrosine metabolism), and beta-Alanine metabolism (Figure 3). In the GO enrichment analysis of the candidate genes for $\mathrm{Fbl}$, among the biological process category, seven metabolic processes (ether metabolic process, polyamine biosynthetic process, amine biosynthetic process, cellular biogenic amine biosynthetic process, RNA splicing via transesterification reactions, RNA splicing via transesterification reactions with bulged adenosine as nucleophile, and polyamine metabolic process) are the most representative groups (Figure 4). For molecular function, the top eight groups include one transporter activity (water transmembrane transporter activity), and seven catalytic activities: oxidoreductase activity (acting on a sulfur group of donors), plus six hydrolase activities (inositol tetrakisphosphate phosphatase activity, inositol trisphosphate phosphatase activity, inositol phosphate phosphatase activity, galactosidase activity, CoA hydrolase activity, and acyl-CoA hydrolase activity). In the cellular components, the highly representative groups are spliceosomal complex, proteasome complex, cell-cell junction, and cell junction (Figure 4). 
A. trait $\mathrm{Ph}(p<0.05)$

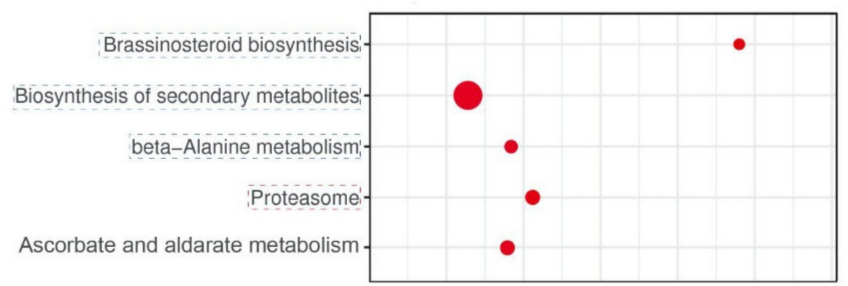

B. trait Fbl $(p<0.05)$

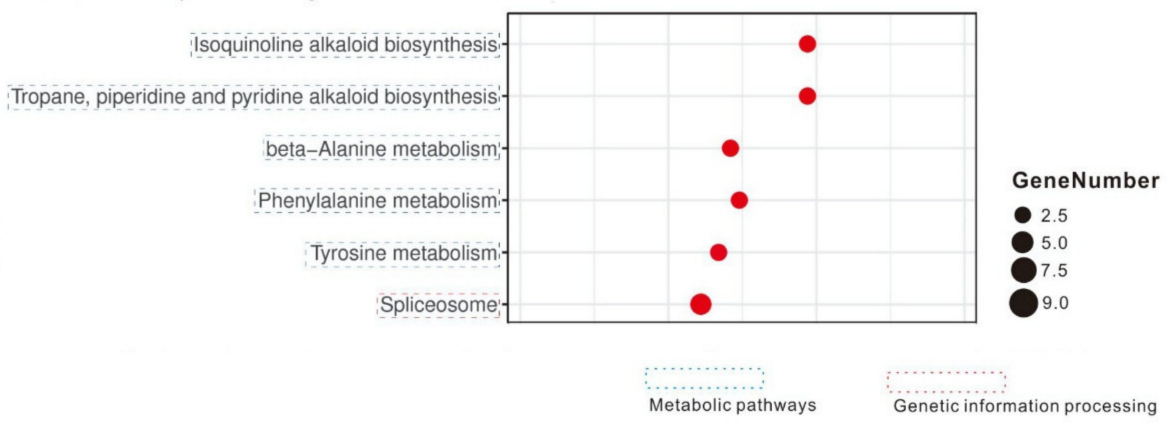

Figure 3. The KEGG pathway enrichment scatter plots of the candidate genes for $\mathrm{Ph}(\mathbf{A})$ and $\mathrm{Fbl}(\mathbf{B})$. Only significant pathways are shown here. The number of candidate genes is indicated by the size of a circle.

A

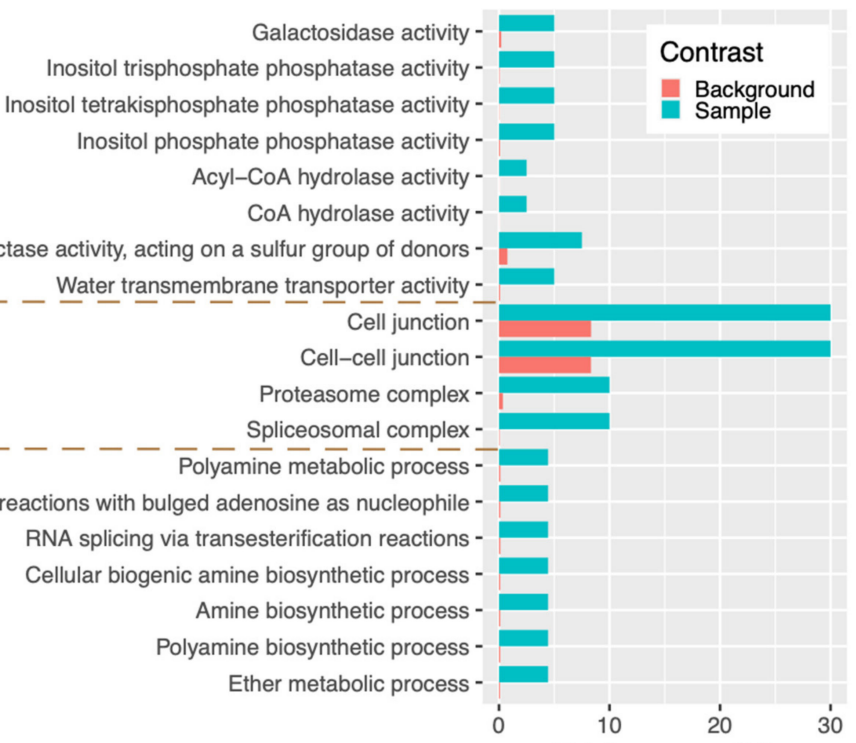

Fbl: frequency (\%)

B

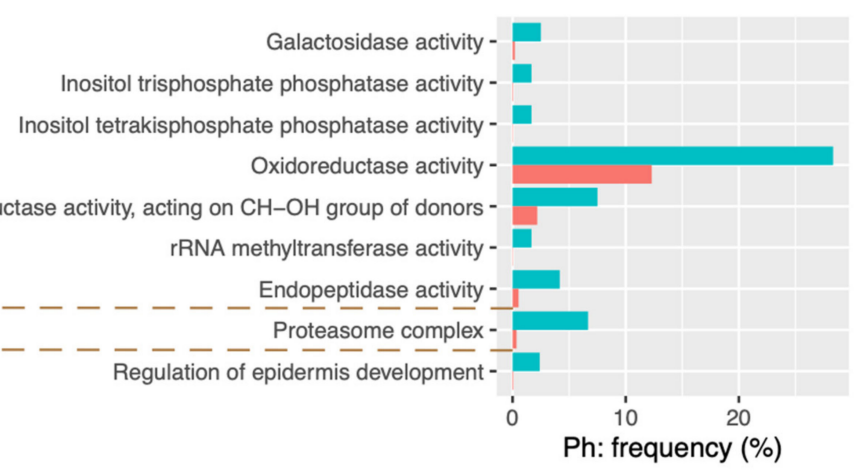

Figure 4. GO terms of candidate genes significantly enriched for $\mathrm{Fbl}(\mathbf{A})$ and $\mathrm{Ph}(\mathbf{B})$. The shown gene frequencies for each GO term are either among the candidate genes (blue) or among the background gene database (orange). 


\section{Discussion}

The cultivated peanut is an important oilseed crop and it is widely cultivated across warm temperate, subtropical, and tropical areas [1,3]. Peanut originated in South America, and from there it has been spread around the world, and in order to adapt to its new and diverse environments, peanut evolved both genetically and phenotypically. In the current study, we have studied 159 peanut accessions, which encompass rich genetic variations by including most of the Chinese peanut landraces [23]. Based on the collected genotypic and phenotypic data, genome-wide association analyses have been performed. The identified candidate genomic regions together with those from earlier QTL analyses are combined in the present study in order to help gain a more comprehensive understanding of the underlying genetic basis for plant height-related traits.

The plant height-related traits are quantitative traits that depend on the cumulative actions of multiple genes and environments. Here, in the present study, we have focused on three plant-height related traits (Ph: plant main stem height, Fbl: the first branch length, and Nsk: the number of nodes on the main stem) and had good control of the environmental factors during both experiments and data analyses. We find significantly associated SNPs for two of the studied traits (Ph and Fbl), KEGG and GO analyses of the genes from these regions together with those from twelve earlier QTL/GWAS studies for the same traits show that both $\mathrm{Ph}$ - and $\mathrm{Fbl}$-associated genes are enriched in the biosynthesis of secondary metabolites. Secondary metabolites are usually specific to a narrow arrangement of species, defining the diverse "personalities" of different organisms, caffeine, alkaloids, flavonoids, and tannins represents good examples of secondary metabolites in plants [24]. Secondary metabolites are traditionally believed not to play key roles in plants' primary life (like development, growth, and reproduction), however, they are well known for their enormous genetic and chemical diversity both across and within species, which definitely suggests the importance of their presence [24-27]. Popular speculation about secondary metabolites' importance is their involvement in the ecological interactions of plants with the highly challenging and varying environments (both biotic and abiotic), which is also supported by many studies [26,28]. For example, when exposed to drought conditions, alkaloids production has been shown to be enhanced in plants for coping with the oxidative stress caused by water shortage $[29,30]$. Generally, under stresses, the elevated secondary metabolite production requires resources to be reallocated from other important activities such as growth $[26,28,31]$. Therefore, it may be not surprising for us to find secondary metabolite biosynthetic genes being highly represented within the genomic regions that are associated with peanut height-related traits, and this overrepresentation may suggest that it is common for peanuts to face intensive environmental stresses even under well-controlled experimental conditions.

Candidate genes for $\mathrm{Ph}$ and $\mathrm{Fbl}$ are also both highly represented in beta-Alanine metabolism. As the precursor of CoA, beta-alanine is important for the metabolisms of phospholipids and fatty acids, as well as for the tricarboxylic acid cycle. It has also been shown that beta-alanine can protect plants from a variety of abiotic and biotic stresses (such as extreme temperature, drought, and heavy metal) [19,32,33], perhaps, apart from the above-mentioned biotic stresses, globally elevated summer temperatures as a consequence of climate change may represent another source of stress for peanut. In addition, $\mathrm{Ph}$ - and Fbl-associated genes tend to be both involved in some basic and essential catalytic activities (hydrolase and oxidoreductase activities).

In addition to the above-mentioned common pathways/activities for $\mathrm{Ph}$ and $\mathrm{Fbl}$, there are also trait-specific enrichments that are mostly in basic processes, pathways, or complexes, which are supposed to be crucial for plant development and growth. For example, $\mathrm{Ph}$-associated genes are also enriched in brassinosteroids biosynthesis, ascorbate and aldarate metabolism, and regulation of epidermis development $[33,34]$. For Fbl, its candidate genes are also enriched in amino acid metabolism, ether metabolic process, amine biosynthetic process, RNA splicing, polyamine metabolic process, and water transmembrane transporter activity. 
However, the interpretation of both GO and KEGG results need to be cautious, considering the fact that not all predicted peanuts proteins can be mapped to a known GO term or KO ID and it is common for one enzyme to be involved in several different pathways or processes [35]. Therefore, future investigation is needed to verify our findings.

\section{Materials and Methods}

\subsection{Plant Materials}

In this study, a total of 159 peanut accessions were collected from 29 Chinese provinces, representing the peanut cultivation areas in China. These accessions belonged to two subspecies and four botanical varieties: 89 A. hypogaea ssp. hypogaea (66 var. hypogea and 23 var. hirsuta), 61 A. hypogaea ssp. fastigiata (51 var. vulgaris and 10 var. fastigiata), and nine irregular types (Figure S1; Table S1). The irregular types usually were the hybrids among the four botanical varieties $[23,36]$.

\subsection{Phenotypic Statistics}

All peanut accessions were planted at three different locations (Dongying, Juxian, and Laixi) in China during 2013, 2014, and 2016. Each accession was represented by 34-40 plants that were grown in a two-row plot $(5.00 \mathrm{~m}$ long and $0.80 \mathrm{~m}$ wide). Three plant height-related traits were measured for each accession: main stem height (Ph), the number of nodes on the main stem (Nsk), and the first branch length (Fbl). Each trait for each harvested accession per location and per year was repeatedly measured five times. To minimize environmental effects, a mixed linear model was built for each trait (as response variable) using the R version 3.6.1 function "lem4" (https:/ / www.r-project. $\mathrm{org} /$, (accessed on 5 July 2019)). Within this model, all included explanatory variables had random effects: the nine combinations between growing years and locations were considered as environmental blocks, the five repeats for each studied plant accession were nested within a block, the peanut accessions, and the interaction between accession and environmental blocks. From the constructed model, the BLUP (Best Linear Unbiased Prediction) estimates of the random genetic effect of peanut accessions on the trait that was on focus were extracted, and it was these BLUP values that would be used (as response variable) later on in the GWAS analyses to study their associations with the identified high-quality SNPs (see below) [37]. The correlation coefficients of each pair of the analyzed traits were calculated with the R version 3.6.1 function "cor" (https: / / cran.r-project.org/ bin/windows/base/, (accessed on 5 July 2019)), and the broad-sense heritability ( $\left.H^{2}\right)$ for each trait was estimated using the R package "lem4".

\subsection{Genome-Wide SNP Collection}

High-quality NGS sequence reads acquired via a genotyping by sequencing (GBS) approach for the 159 studied peanut accessions were downloaded from the Sequence Read Archive database (SRA accession: PRJNA525244) [18]. The acquired high-quality reads were then mapped onto a peanut reference genome (https:/ / www.peanutbase.org/data/ public/Arachis_hypogaea/Tifrunner.gnm1.KYV3/ (accessed on 5 July 2019)) using BWA v0.6.2 (-t $4-\mathrm{M}-\mathrm{k} 32-\mathrm{r} 1-\mathrm{c} 1$ ) [38]. Variant calling was performed using the GATK's Unified Genotyper (https: / / software.broadinstitute.org/gatk (accessed on 5 July 2019)). The identified variants were filtered to reduce the false-positive errors using GATK Variant Filtration. To im-prove variation quality, the following criteria were adopted: (i) quality score $>20$; (ii) coverage depth $>3$ fold; (iii) missing ratio within each population $<80 \%$; (iv) a global minor allele frequency $(\mathrm{MAF})>0.05$. The genic and intergenic locations of all high-quality variants (including SNPs) were determined using ANNOVAR [39].

\subsection{Genome-Wide Association Study Analysis}

Genome-wide association study (GWAS) of the three plant height-related traits based on the acquired high-quality SNPs were conducted using TASSEL v5.0 $[37,38]$ Four different models were tried for each trait, including two general linear models: GLM, $Y=X a+e$; 
GLM(G), $Y=X a+Q b+e$, and two Mixed Linear Model: $\operatorname{MLM}(\mathrm{K}), Y=X a+K u+e$; $\operatorname{MLM}(\mathrm{GK}), Y=X a+Q b+K u+e$, where $Y$ denoted the phenotype (represented by the BLUP values as estimated above) and $X$ the genotype at each SNP locus, $Q$ represented population structure estimated by Admixture and $K$ was the relationship between samples (i.e. kinship coefficients). For each trait, the best model out of the tested four based on the Q-Q plot was accepted as the final model. We used a $p$-value of $10^{-5}$ or less to establish the significance. The candidate genome regions that might contain genes responsible for the studied plant height-related traits were the most strongly associated SNPs (hereafter referred to as peak SNPs) plus a 100kb-long genomic region that were centered on the peak SNPs $[18,40-42]$. The matrix of pairwise kinship coefficients among the studied accessions was calculated using the software SPAGeDi v1.5 [43].

\subsection{A Literature Survey of QTLs for Peanut-Height Related Traits}

To look for more candidate genes underlying peanut height-related traits, we carried out a thorough literature survey of related QTL/GWAS studies. All genes within the candidate genomic regions that were identified by both the present GWAS analyses and earlier studies were considered as candidate genes for the related traits. Both Gene Ontology (GO) enrichment analysis, and KEGG (Kyoto Encyclopedia of Genes and Genomes pathway database) pathway enrichment analysis was carried out on the candidate genes using the OmicShare tools, a free online platform for data analysis (www.omicshare.com/tools, (accessed on 5 July 2020)). To accommodate the multiple testing problem in the enrichment analysis, Benjamini-Hochberg adjusted $p$-values were calculated [44].

Supplementary Materials: The following are available online at https://www.mdpi.com/article/ 10.3390/plants10061058/s1. Figure S1: Geographic distribution of the analyzed peanut accessions. Each accession is displayed as a dot. Different colors stand for the different botanical varieties. (NOTE: The map used here represents only part of China). Figure S2: The overall frequency distributions for the three studied plant height-related traits, included the main stem (Nsk), the first branch length $(\mathrm{Fbl})$, and plant main stem height $(\mathrm{Ph})$. Figure S3: The frequency distribution for the number of nodes on the main stem (Nsk) in different years at different locations. Figure S4: The frequency distribution for the first branch length $(\mathrm{Fbl})$ in different years at different locations. Figure S5: The frequency distribution for plant main stem height $(\mathrm{Ph})$ in different years at different locations. Figure S6: The correlation between the three studied plant height-related traits (Fbl, Ph, Nsk). Figure S7: Manhattan plots and Q-Q plots from the GWAS analyses of two plant height-related traits ( $\mathrm{Ph}$ and $\mathrm{Fbl}$ ). For each trait, the Q-Q plots from four different statistical models are shown on the right. The shown Manhattan plots are from the best model. The black arrows pointed to 11 peak SNPs that are associated with Ph and/or Fbl. The significance level is $-\log 10(0.05 / 17117)=5.1$ (the red horizontal line). Table S1: Peanut cultivars' code numbers and sources Table S2: A summary of the acquired SNPs and InDels in different genic and intergenic regions. Table S3: The correlation among the plant height-related traits. Table S4: Information on earlier identified QTLs for Ph and Fbl and their physical locations. Table S5: A summary of the specific/common candidate genomic regions for $\mathrm{Ph}$ and $\mathrm{Fbl}$ that are acquired from both the present and earlier studies. Table S6: Information on the annotated genes from the candidate genome regions for $\mathrm{Ph}$ and $\mathrm{Fbl}$ that are acquired from both the present and earlier studies.

Author Contributions: Conceptualization, S.S. and J.W.; methodology, J.W.; software, C.Y. (Caixia Yan); validation, J.W., D.S., and Y.L.; formal analysis, C.Y. (Cuiling Yuan); investigation, Q.S. and Y.M.; resources, C.Y. (Cuiling Yuan) and H.C.; data curation, J.W. and D.S.; writing-original draft preparation, J.W.; writing — review and editing, J.W., Y.L., C.L., and S.S.; visualization, X.Z.; supervision, Y.L., C.L., and S.S.; project administration, C.L. and S.S.; funding acquisition, S.S., D.S., and X.Z. All authors have read and agreed to the published version of the manuscript.

Funding: This work was supported by Taishan Scholars Project (ts201712080), Key Research and Development Project of Shandong Province (2020LZGC001), National Natural Science Foundation of China (31800164, 31901506), and Agro-industry Technology Research System of Shandong Province (SDAIT-04-02). The funders had no role in study design, data collection, and analysis, decision to publish, or preparation of the manuscript. 


\section{Data Availability Statement: Not applicable.}

Acknowledgments: The authors would like to acknowledge Xiangdong Liu from Shandong Peanut Research Institute for their fieldwork and phenotypic data collection of this paper.

Conflicts of Interest: The authors declare no conflict of interest. The funders had no role in the design of the study; in the collection, analyses, or interpretation of data; in the writing of the manuscript, or in the decision to publish the results.

\section{References}

1. Bertioli, D.J.; Cannon, S.B.; Froenicke, L.; Huang, G.; Farmer, A.D.; Cannon, E.K.; Liu, X.; Gao, D.; Clevenger, J.; Dash, S.; et al. The genome sequences of Arachis duranensis and Arachis ipaensis, the diploid ancestors of cultivated peanut. Nat. Genet. 2016, 48, 438-446. [CrossRef] [PubMed]

2. Bertioli, D.J.; Jenkins, J.; Clevenger, J.; Dudchenko, O.; Gao, D.; Seijo, G.; Leal-Bertioli, S.C.M.; Ren, L.; Farmer, A.D.; Pandey, M.K.; et al. The genome sequence of segmental allotetraploid peanut Arachis hypogaea. Nat. Genet. 2019, 51, 877-884. [CrossRef] [PubMed]

3. Stalker, H.T. Utilizing wild species for peanut improvement. Crop. Sci. 2017, 57, 1102-1120. [CrossRef]

4. Yu, S.L. Peanut Genetics and Breeding in China; Shanghai Science and Technology Press: Shanghai, China, $2011 ;$ pp. 11-13.

5. Varshney, R.K.; Nayak, S.N.; May, G.D.; Jackson, S.A. Next-generation sequencing technologies and their implications for crop genetics and breeding. Trends Biotechnol. 2009, 27, 522-530. [CrossRef] [PubMed]

6. Varshney, R.K.; Mohan, S.M.; Gaur, P.M.; GangaRao, N.; Pandey, M.K.; Bohra, A.; Sawargaonkar, S.L.; Chitikineni, A.; Kimurto, P.K.; Janila, P.; et al. Achievements and prospects of genomics-assisted breeding in three legume crops of the semi-arid tropics. Biotechnol. Adv. 2013, 31, 1120-1134. [CrossRef]

7. Khedikar, Y.; Pandey, M.K.; Sujay, V.; Singh, S.; Nayak, S.N.; Klein-Gebbinck, H.W.; Sarvamangala, C.; Mukri, G.; Garg, V. Identification of main effect and epistatic quantitative trait loci for morphological and yield-related traits in peanut (Arachis hypogaea L.). Mol. Breeding 2018, 38, 1-12.

8. Cheng, L.Q.; Tang, M.; Ren, X.P.; Huang, L.; Chen, W.; Li, Z.; Zhou, X.; Chen, Y.; Liao, B.; Jiang, H. Construction of genetic map and QTL analysis for mian stem height and total branch number in peanut. Acta Agrono. Sin. 2015, 41, 979-987. [CrossRef]

9. Huang, L.; He, H.; Chen, W.; Ren, X.; Chen, Y.; Zhou, X.; Xia, Y.; Wang, X.; Jiang, X.; Liao, B.; et al. Quantitative trait locus analysis of agronomic and quality-related traits in cultivated peanut (Arachis hypogaea L.). Theor. Appl. Genet. 2015, 128, 1103-1115. [CrossRef]

10. Lan, X.L.; Tang, Z.X.; Xu, R. Analysis of gray gorrelation between yield and major agronomic traits of peanut in Fujian Province. Acta Agric. Jiangxi 2011, 23, 61-63. [CrossRef]

11. Yin, D.M.; Shang, M.Z.; Cui, D.Q. Studies on genetic analysis of major agronomic characters in peanut. Chin. Agric. Sci. Bull. 2006, 22, 261-265.

12. Kover, P.X.; Valdar, W.; Trakalo, J.; Scarcelli, N.; Ehrenreich, I.M.; Purugganan, M.D.; Durrant, C.; Mott, R. A Multiparent Advanced Generation Inter-Cross to Fine-Map Quantitative Traits in Arabidopsis thaliana. PLoS Genet. 2009, 5, e1000551. [CrossRef] [PubMed]

13. Zhang, S.Z.; Qiu, J.L.; Miao, H.R.; Yang, W.Q.; Zhao, L.B.; Song, X.; Pan, L.J.; Zhang, Z.M.; Hu, X.H.; Chen, J.; et al. QTL mapping for main stem height, first branch length and branch number based on a high-density linkage map in peanut (Arachis hypogaea L.). J. Peanut Sci. 2019, 48, 1-9.

14. Lv, J.; Liu, N.; Guo, J.; Xu, Z.; Li, X.; Li, Z.; Luo, H.; Ren, X.; Huang, L.; Zhou, X.; et al. Stable QTLs for Plant Height on Chromosome A09 Identified from Two Mapping Populations in Peanut (Arachis hypogaea L.). Front. Plant Sci. 2018, 9, 684. [CrossRef] [PubMed]

15. Chen, W.-G.; Guo, J.-B.; Xu, Z.-J.; Yu, B.-L.; Qiu, X.-K.; Huang, L.; Song, Y.-B.; Chen, Y.-N.; Zhou, X.-J.; Luo, H.-Y.; et al. QTL Mapping for Shelling Percentage and Plant Height in Cultivated Peanut (Arachis hypogaea L.). Acta Agron. Sin. 2018, 44, 1142-1151. [CrossRef]

16. Li, Y.; Li, L.; Zhang, X.; Zhang, K.; Ma, D.; Liu, J.; Wang, X.; Liu, F.; Wan, Y. QTL mapping and marker analysis of main stem height and the first lateral branch length in peanut (Arachis hypogaea L.). Euphytica 2017, 213, 57. [CrossRef]

17. Li, Y.J. QTL Analysis for Plant Height, Total Branching Number and Pod Traits in Peanut (Arachis hypogaea L.). Master's Thesis, Shandong Agricultural University, Shandong, China, 2016.

18. Wang, J.; Yan, C.X.; Li, Y.; Li, C.; Zhao, X.; Yuan, C.; Sun, Q.; Shan, S. GWAS discovery of candidate genes for yield-related traits in peanut and support from earlier QTL mapping studies. Genes 2019, 10, 803. [CrossRef]

19. De Abreu, I.N.; Mazzafera, P. Effect of water and temperature stress on the content of active constituents of Hypericum brasiliense Choisy. Plant Physiol. Biochem. 2005, 43, 241-248. [CrossRef]

20. Song, W.; Forderer, A.; Yu, D.L.; Chai, J.J. Structural biology of plant defense. New Phytol. 2021, 229, 692-711. [CrossRef]

21. Liu, P.-L.; Du, L.; Huang, Y.; Gao, S.-M.; Yu, M. Origin and diversification of leucine-rich repeat receptor-like protein kinase (LRR-RLK) genes in plants. BMC Evol. Biol. 2017, 17, 47. [CrossRef]

22. Chakraborty, S.; Nguyen, B.; Wasti, S.D.; Xu, G. Plant Leucine-Rich Repeat Receptor Kinase (LRR-RK): Structure, Ligand Perception, and Activation Mechanism. Molecules 2019, 24, 3081. [CrossRef]

23. Shan, S.H.; Yan, C.X. Key Germplasms of Chinese Peanut Landraces; China Agriculture Press: Beijing, China, 2018. 
24. Zhang, H. Construction of Core Collections and Genetic Diversity Analysis of Arachis hypogaea L., Cultivars in China. Master's Thesis, ZhongKai University of Agriculture and Engineering, Guangzhou, China, 2013.

25. Ncube, B.; Van Staden, J. Tilting Plant Metabolism for Improved Metabolite Biosynthesis and Enhanced Human Benefit. Molecules 2015, 20, 12698-12731. [CrossRef] [PubMed]

26. Benderoth, M.; Textor, S.; Windsor, A.; Mitchell-Olds, T.; Gershenzon, J.; Kroymann, J. Positive selection driving diversification in plant secondary metabolism. Proc. Natl. Acad. Sci. USA 2021, 103, 9118-9123. [CrossRef]

27. Kroymann, J. Natural diversity and adaptation in plant secondary metabolism. Curr. Opin. Plant Biol. 2011, 14, 246-251. [CrossRef] [PubMed]

28. Rhodes, M.J.C. Physiological roles for secondary metabolites in plants: Some progress, many outstanding problems. Plant Mol. Biol. 1994, 24, 1-20. [CrossRef]

29. Isah, T. Stress and defense responses in plant secondary metabolites production. Biol. Res. 2019, 52, 39. [CrossRef]

30. Selmar, D.; Kleinwächter, M. Stress enhances the synthesis of secondary plant products: The impact of stress-related overreduction on the accumulation of natural products. Plant Cell Physiol. 2013, 54, 817-826. [CrossRef] [PubMed]

31. Yahyazadeh, M.; Meinen, R.; Hänsch, R.; Abouzeid, S.; Selmar, D. Impact of drought and salt stress on the biosynthesis of alkaloids in Chelidonium majus L. Phytochemistry 2018, 152, 204-212. [CrossRef]

32. Parthasarathy, A.; Savka, M.A.; Hudson, A.O. The Synthesis and Role of $\beta$-Alanine in Plants. Front. Plant Sci. 2019, 10, 921. [CrossRef]

33. Klapheck, S.; Zopes, H.; Bergmann, L.; Levels, H.-G. Properties and localization of the homoglutathione synthetase from Phaseolus coccineus leaves. Physiol. Plant. 1988, 74, 733-739. [CrossRef]

34. Nolan, T.M.; Vukašinović, N.; Liu, D.; Russinova, E.; Yin, Y. Brassinosteroids: Multidimensional Regulators of Plant Growth, Development, and Stress Responses. Plant Cell 2020, 32, 295-318. [CrossRef]

35. Gest, N.; Gautier, H.; Stevens, R. Ascorbate as seen through plant evolution: The rise of a successful molecule? J. Exp. Bot. 2013, 64, 33-53. [CrossRef]

36. Zhou, Y.; Gao, Y.; Xu, C.; Shen, H.; Tian, Q.; Deng, H.-W. A novel approach for correction of crosstalk effects in pathway analysis and its application in osteoporosis research. Sci. Rep. 2018, 8, 668. [CrossRef]

37. Jiang, H.F.; Duan, N.; Ren, X.P. Descriptors and Data Standard for Peanut (Arachis spp.); China Agriculture Press: Beijing, China, 2006.

38. Li, H.; Durbin, R. Fast and accurate short read alignment with Burrows-Wheeler transform. Bioinformatics 2009, 25, 1754-1760. [CrossRef] [PubMed]

39. Wang, K.; Li, M.; Hakonarson, H. ANNOVAR: Functional annotation of genetic variants from high-throughput sequencing data. Nucleic Acids Res. 2010, 38, e164. [CrossRef] [PubMed]

40. Bradbury, P.J.; Zhang, Z.; Kroon, D.E.; Casstevens, T.M.; Ramdoss, Y.; Buckler, E.S. Tassel: Soft-ware for association mapping of complex traits in diverse samples. Bioinformatics 2007, 23, 2633-2635. [CrossRef]

41. Glaubitz, J.; Casstevens, T.; Lu, F. TASSEL-GBS: A high capacity genotyping by sequencing analysis pipe-line. PLoS ONE 2013, 9, e90346.

42. Zhang, X.; Zhang, J.; He, X.; Wang, Y.; Ma, X.; Yin, D. Genome-Wide Association Study of Major Agronomic Traits Related to Domestication in Peanut. Front. Plant Sci. 2017, 8, 1611. [CrossRef]

43. Hardy, O.J.; Vekemans, X. SPAGeDi: A versatile computer program to analyses spatial genetic structure at the individual or population levels. Mol. Ecol. Resour. 2002, 2, 618-620. [CrossRef]

44. Huang, D.W.; Sherman, B.T.; Lempicki, R.A. Bioinformatics enrichment tools: Paths toward the comprehensive functional analysis of large gene lists. Nucleic Acids. Res. 2009, 37, 1-13. [CrossRef] [PubMed] 